\title{
How Do Interprofessional Healthcare Teams Perceive the Benefits and Challenges of Interdisciplinary Ward Rounds
}

This article was published in the following Dove Press journal: Journal of Multidisciplinary Healthcare

\author{
Victoria Walton' \\ Anne Hogden (D) ${ }^{\prime}$ \\ Janet $C$ Long $\mathbb{D}^{2}$ \\ Julie K Johnson $\mathbb{1 D}^{3}$ \\ David Greenfield (ID)
}

\begin{abstract}
'Australian Institute of Health Service Management, University of Tasmania, Sydney, NSW 20I5, Australia; ${ }^{2}$ Australian Institute of Health Innovation, Macquarie University, Sydney, NSW, Australia;

${ }^{3}$ Centre for Healthcare Studies, Feinberg School of Medicine, Northwestern University, Chicago, Illinois, USA
\end{abstract}

Purpose: Interdisciplinary bedside ward rounds have the capacity to facilitate coordinated interprofessional patient care. To be an effective means of care coordination, clinicians need an explicit understanding of how these rounds contribute to patient care. By identifying benefits and challenges to the effective use of interdisciplinary ward rounds, clinicians create an opportunity to improve interprofessional teamwork, care planning, and coordination of patient care.

Methods: A survey was conducted with frontline professionals in two acute care and two rehabilitation wards from a metropolitan teaching hospital. There were 77 participants, representing medical officers, nurses, and allied health clinicians. Questions examined the perceived benefits and challenges of conducting interdisciplinary ward rounds in their units. Survey findings were coded for meaning and then grouped into themes.

Results: Benefits revealed a desired care delivery model challenged by the complexities of organizational and professional cultures. The themes of "being on the same page", "focusing on patients", and "holistic care planning" underpinned the ideas of collaboration and improved patient-centred care, that is, benefits to patients. Challenges centred on health professionals' time constraints and the coordination of teams to enable participation in rounds. The themes were more distinct, logistical barriers of "time", "workforce", and "care planning".

Conclusion: Overall, clinicians recognise there are greater benefits to IBRs and have a willingness to participate. However, careful consideration is required to introduce and continually achieve the best from IBR as they require changes in organizational context and culture.

Keywords: challenges, benefits, coordination, patient focused care, time factors, communication

\section{Introduction}

Ward rounds have been a pivotal part of traditional hospital life in the planning and delivery of patient care. ${ }^{1}$ Additionally, they provide a platform for health professionals to give and receive clinical education. Different rounding processes combine these goals in specific ways, as reflected by their titles. They include: ward; multidisciplinary; consultant; teaching; post-take; traditional; working; and review of ward. ${ }^{2}$ There is a significant variance in the structure and design of rounds. ${ }^{3}$ Variations can include: the structure and focus of the round, such as for pharmacy or discharge, ${ }^{4}$ the location of the round, such as bedside or corridor; and the names
Correspondence: Victoria Walton Australian Institute of Health Service Management, University of Tasmania, Locked Bag 5052, Alexandria, Sydney NSW 2015, Australia

Tel +6I 2408417814

Email victoria.walton@utas.edu.au 
of rounds can be interchangeable, such as "interdisciplinary" with "multidisciplinary."2

Naming variations associated with different care models may influence rounding processes. Health professionals working in a multidisciplinary team structure work in parallel. That is, each discipline has their own goals for the patient rather than shared goals with the team. ${ }^{5}$ Interdisciplinary care teams work more collaboratively to plan patient goals as a team. ${ }^{5}$

Different rounding processes show variance in multidisciplinary round participants and roles. ${ }^{2}$ Three combinations were identified; medical, nursing, and allied health; medical, nursing, allied health and patient; and medical, nursing and patient. The medical role was described in all studies. The role often took on one of leadership, teaching and decision-making around patient care. The nursing role was the next most commonly described. The roles included patient advocacy, to present patient care, and to a lesser degree, leadership during the round. The role of allied health clinicians, such as speech pathologists, dieticians, social workers, physiotherapists and pharmacists, was not specified in half of all studies that included them. Studies that did specify their role showed they were concerned with discharge planning and medication management when a pharmacist was a participant. The patient role was described as clarifying treatment plans, goals and discharge plans. ${ }^{2}$ The combination of these variances can impact the safety and quality of the care patients receive. ${ }^{6}$

Not surprisingly, health professionals' understanding and experiences of their own ward rounds, within and between disciplines and specialities, vary across rounding processes. ${ }^{7}$ In the acute settings studied, nursing and allied health clinicians participated in multidisciplinary bedside rounds. By contrast, medical officers did not identify multidisciplinary rounding processes. This differed from the rehabilitation settings studied, where representatives from each health profession agreed that the rounds they undertook were multidisciplinary rounds. ${ }^{7}$ In short, healthcare teams can, and do, participate in rounds without a shared understanding of the process or role requirements.

Awareness amongst healthcare team members of their roles and responsibilities in rounds enables a more collaborative approach to care planning. ${ }^{8}$ Interdisciplinary bedside rounds (IBRs) are known to improve team collaboration and satisfaction, ${ }^{4,9}$ and coordination. ${ }^{3}$ Specific benefits include: improved interprofessional communication; greater awareness of patient care issues; improved team communication about the patient care plan; inclusion of the patient in decision-making; and teaching opportunities. ${ }^{3,10}$ IBRs have been shown to improve the quality and safety of the care delivery through reduced mortality rates and long-term morbidity. ${ }^{11}$

IBRs, however, do not guarantee a collaborative approach to care delivery as health professionals can be restrained by the need to hold onto boundaries and knowledge. ${ }^{11}$ Challenges include: time constraints; coordination of clinicians' availability; and the length of time taken for discussion. ${ }^{3,10}$ Introducing or consolidating interdisciplinary bedside ward rounds may be challenging as they require clinicians to make a cultural shift from working in silos to working collaboratively. Support at an organizational level is also necessary to facilitate this shift. $^{12,13}$

The variance in health professional representation during IBRs, combined with disconnection in round identification, leads us to question if healthcare teams are "on the same page" when organizing and delivering care. Hence, we sought to investigate medical officers, nurses and allied health professionals' perceptions of the benefits and challenges of IBRs. Examining the complex issue of IBRs, we aimed to uncover professional's understanding and practice, across disciplines and specialities, and the implications of these on care coordination. In this paper, we use the term interdisciplinary to describe health professionals from medial, nursing and allied health professions collaborating to plan patient care. IBRs refer to rounds that are undertaken at the patient's bedside. Allied Health disciplines involved in this study are: physiotherapy, speech pathology, dietetics, neurophysiologist, and occupational therapy.

\section{Method}

\section{Setting}

The study setting was a teaching hospital located in metropolitan Sydney, Australia. Adult inpatient services are provided in medicine, surgery, critical care, and rehabilitation. Clinicians working in four wards in two specialties acute medicine and rehabilitation services - were invited to participate in the study. IBRs were not routinely undertaken within either speciality.

\section{Ethics}

Ethics approval for the study was given by a metropolitan local health district research ethics committee prior to the 
commencement of the research. The approval reference is LNR.13.HAWKE.433.

\section{Research Design}

Between March 2014 and March 2015, a qualitative study was carried out. A paper-based survey was distributed to clinicians from all seniority levels across medical, nursing, and allied health professions. The survey was purposely designed for the study. Questions were informed by literature $^{2}$ and the investigating team members' industry experience. Participants were asked to consider the benefits and challenges of interdisciplinary bedside rounds, to identify up to five issues for each theme. The tool provided space for up to five issues documented in free text. Additionally, at the end of each question, a statement saying, "No benefits" and "No disadvantages" were provided as an answer option (Figure 1). Participants were provided with the definition at the start of the survey. ${ }^{2}$ No examples of benefits or challenges were provided as prompts.

The survey was pre-tested within an interdisciplinary team with equivalent experience and context within the same local health district, to assess question understanding and test our analysis technique. No changes were made to the original format.

\section{Data Collection}

Paper-based surveys were distributed directly to clinicians by a member of the research team. Both verbal and written instructions were provided. Written consent was obtained

An interdisciplinary ward round is when 1 .

the specific medical, nursing and allied health clinicians involved in a patient's care meet with the patient and along with the patient summarise and plan the nest steps in the patients care.

What are the benefits to having a multidisciplinary ward round? (list up to five)

What are the disadvantages to having a 1 . multidisciplinary ward round? (list up to five)

2.

3.

4.

5.

No disadvantages 
prior to survey. Participation was voluntary, and supported by ward managers and health professional directors, who facilitated clinician participation by providing rooms and times so that the survey could be completed. The researcher visited the wards to distribute the surveys and waited to collect them. The survey took approximately 15 mins to complete and was conducted during a time nominated by participants that did not disturb clinical work.

\section{Analysis}

Data were entered into an MS Excel file. Each participant was assigned a code, and any identifiable text was deidentified during the data entry stage. A thematic analysis was adopted. This allowed researchers to become more familiar with the study for responses and word diversity to be considered within the context of the whole text. ${ }^{14}$ Analysis was guided by the Schwandt, Lincoln and Guba ${ }^{15}$ framework for analysis. Responses were coded and grouped into similar concepts which then became the basis for the overarching themes (VW). Linking concepts lead to the development of sub-themes using key elements of each to support them. Themes and sub-themes were compared between specialty, and within and across disciplines. These enabled researchers to identify relationships between challenges and barriers within different clinician cohorts. Codes and themes were discussed with the research team to ensure a common understanding and agreement. Any disagreements were talked through within the team. Implications and meaningful application of the findings to the practical healthcare environment were discussed within the team. Throughout the findings, participant quotes were extracted to support the themes. Participants were coded by professional group: medical officer (MO); nursing $(\mathrm{N})$; and allied health professional (AH).

\section{Findings}

\section{Response Rate}

Eighty-three health professionals were approached. Seventy-seven participants completed the survey, resulting in a $93 \%$ response rate. Acute medicine respondents totalled 26 (34\% of all respondents). Rehabilitation respondents totalled 51 (66\% of all respondents). The greatest number of surveys were completed by nurses $(n=46)$, followed by health-allied clinicians $(n=20)$, and medicine completed the fewest $(\mathrm{n}=11)$. Seventy-one participants completed the questionnaire on the benefits of an interdisciplinary bedside round (11/11 medical officers; 41/46 nurses; and 19/20 allied health professionals). Free text responses varied between bullet point responses and short paragraphs.

\section{Benefits of Interdisciplinary Bedside Ward Rounds}

A total of 268 individual benefits were identified by participants. These were categorised into three overarching and interrelated themes (Table 1). Within these, seven subthemes and 10 key elements were identified. No participants chose the "no advantage" option.

\section{Theme One: Being on the Same Page}

The most common comments provided by respondents related to "being on the same page". Health professionals most frequently identified teamwork as a benefit of interdisciplinary rounds. All health professions identified benefits that described interdisciplinary rounds as building more cohesive teamwork. Specifically, medical officers noted this may reduce team conflict and provide a greater understanding of the patient's care. Improved

Table I Benefits of Interdisciplinary Ward Round

\begin{tabular}{|c|c|c|}
\hline Themes & Sub-Themes & Key Elements \\
\hline Being on the same page & $\begin{array}{l}\text { Effective communication } \\
\text { Efficient workflow }\end{array}$ & $\begin{array}{l}\text { - Direct communication between team members } \\
\text { - Developing a more cohesive healthcare team } \\
\text { - Medical officers more accessible to other clinicians } \\
\text { - Improved teamwork }\end{array}$ \\
\hline Focusing on patients & $\begin{array}{l}\text { Patient satisfaction } \\
\text { Access to information }\end{array}$ & $\begin{array}{l}\text { - Patients gain confidence when they see the team working together } \\
\text { - Enables patient and family to be better informed of their care } \\
\text { - Individualised care }\end{array}$ \\
\hline Holistic care planning & $\begin{array}{l}\text { Patient flow } \\
\text { Care planning clarity } \\
\text { Shared contribution to care planning }\end{array}$ & $\begin{array}{l}\text { - Discharge planning } \\
\text { - Focus is on interdisciplinary information not just medical } \\
\text { - Plans are current and relevant }\end{array}$ \\
\hline
\end{tabular}


teamwork was facilitated by: awareness of individual team member's roles and responsibilities; understanding individual clinician's progress with patients; and sharing knowledge within the wider team.

Understand each other's roles better and brings better team-work. [N42]

Builds team camaraderie. [N44]

Teamwork, get to know other clinicians. [AH3]

Clinicians stated that interdisciplinary rounds would improve communication both within the healthcare team, but also between the team and patient. "Being on the same page" was a phrase all participant groups used in their response. Medical officers defined this as receiving "real time" information about the patient. Nurses described being on the same page as providing consistent expectations for patients. For allied health, being on the same page enabled patients to speak directly with the whole healthcare team rather than multiple clinicians at different times. This was further described as improving patient satisfaction, thereby reducing patient complaints. Allied health clinicians stated they could more easily raise concerns with medical officers as they could speak to them directly.

Nursing staff and allied health able to express concerns to patient and medical officers. [AH9]

Everyone is on the same page and expectations are clear. [N25]

Saves time as the information is relayed then and there. [M7]

The opportunity to have face-to-face communication was identified by both nursing and allied health clinicians. It was considered more reliable than written documentation.

Often progress notes are not sufficient as a method of passing on information. [N37]

It saves me from finding out medical orders written in the medical records at the end of my shift. [AH4]

Workflow efficiencies were described in terms of timesaving processes. Meeting with the whole healthcare team and being able to talk directly to both colleagues and patients reduced time spent on following up information. Participants reported that clarification of orders could be addressed at the time they are made and with the person making the order. Nursing and allied health clinicians noted that this reduces redundant communication with team members and reduces repeating information while seeking clarification from different team members. From the patient's perspective, IBRs reduce repetition of questions being asked of them.

Saves times to chase up doctors. [N2]

Things are not being repeated over and over again. There is no confusion with what has been ordered or discussed. [N24]

Reduction in over communicating to other team members of the MDT. [N29]

Improves efficiency - don't have to chase for information. [A4]

The patient doesn't need to repeat their wishes to individual team members during one to one sessions. [A18]

\section{Theme Two: Focusing on Patients}

"Focusing on patients" was described by all health professionals from the perspective of the patient and how the patient would benefit. All health disciplines reported patients feeling more cared for if reviewed by the interdisciplinary team together. Medical, nursing and allied health all believed patients would have more confidence if they saw the whole team working together.

Good for the patient to see the team working together. [N1]

Provides the patient with more confidence when they see the team working together. [AH6]

Can solve patient's concerns immediately as everyone is present in ward round. [M11]

Medical officers said this would allow for more than just medical issues to be addressed and would give a greater perspective on the patient's overall progress. Nursing and allied health supported this, indicating that when a complete picture of a patient is provided, goal setting becomes more patient centred. Patients and family members are better informed as they receive information from all team members, which allows any issues or questions to be addressed together. Building on this, allied health clinicians perceived that identified rounds that are held at the same time each day provide more consistency and opportunity for involvement.

More ideas for patient care. [M5] 
Consistency for staff/families/patients/services that team meets and plans at same time every day. [AH5]

Involvement of patients in their care was valued by all disciplines. Having relevant health providers together enabled patients to participate in goal setting and individualised their care. Nursing and allied health agreed these empowered patients to be active participants in their care. Medical and nursing expanded on this, stating that patients who are more actively involved are more likely to be compliant with their care planning and recommendations.

Patient's feel empowered and confident as they are part of the process and thus are more likely to be compliant. [N13]

Patients are directly involved in discussion of goals and treatment. [AH18]

Nursing and allied health both identified patient advocacy as a key element of providing patient-focused care. When present during the round, participants could facilitate discussion between patients and medical officers, especially if a patient was uncomfortable communicating with doctors.

Advocate for patients who may not feel comfortable speaking directly to medical staff. [AH4]

\section{Theme Three: Holistic Care Planning}

"Holistic care planning" elements were nominated by all respondents. While this is closely linked to the previous theme, holistic care planning key findings related to the outcomes and process of providing patient-focused care. All health professional disciplines identified common care planning goals as benefitting themselves, by being more informative and more time-efficient. Medical officers reported access to supplementary information on patients would assist with care planning.

Patient is seen at centre of care and more holistic. [N29]

Addressing not just the medical issues of the patient but also their functioning and preparedness for home environment. [M5]

Holistic view of how the patient is managing which equates to more effective care. [AH4]

Nursing perceived clearer care plans as a benefit. Review of the current care plans by all care providers facilitated proactive interdisciplinary care planning that can be understood by all involved. Similarly, both nurses and allied health clinicians stated they could be involved in formulating care plans.

I can provide information to the patient's care team that helps formulate a plan. [AH6]

Clear pathways. [N11]

...know the most updated patient's condition and to facilitate discharge plans and treatment. [AH8]

Medicine, nursing and allied health clinicians considered that improved patient flow could stem from interdisciplinary rounds. Similarly, discharge plans could be facilitated by rounds through improved care planning, to ensure everyone was working towards the same discharge plan.

All on same page for discharge planning - patient aware too. $[\mathrm{AH} 7]$

Improved discharge planning - leads to decreased length of stay. [M9]

\section{Challenges of Interdisciplinary Bedside Ward Rounds}

A total of 129 individual challenges of interdisciplinary rounds were identified by respondents. These were categorised into three overarching themes (Table 2). Within these, nine subthemes and 10 key elements were identified. Additionally, 17 respondents ( 3 medical officers and 14 nurses) indicated no disadvantages to interdisciplinary rounds.

\section{Theme One: Time}

Health professionals identified "time" as the most significant challenge to undertaking interdisciplinary rounds. Although our survey did not specify which patients would be reviewed on an interdisciplinary round, from their answers, participants indicated an assumption that all patients would be reviewed at every round. Time involved four elements. First, the length of time a round could take if all patients were reviewed daily. The main factors contributing to this were lengthy discussions due to multiple clinical opinions. There were concerns that if the round was not well managed, the time taken to complete it could be extensive. Patients and family were perceived to potentially contribute to a lengthier round by wanting more input, due to having the team all present.

Take a lot of time to discuss patient care individually. [N5]

Time consuming - often 25 patients to see every day. [M6] 
Table 2 Challenges of Interdisciplinary Bedside Ward Rounds

\begin{tabular}{|c|c|c|}
\hline Themes & Sub-Themes & Key Elements \\
\hline Time & $\begin{array}{l}\text { Takes time away from providing care for patients } \\
\text { The time required to complete the ward round } \\
\text { Parallel processes }\end{array}$ & $\begin{array}{l}\text { - Takes clinical time from other patients } \\
\text { - Multiple clinical opinions will increase discussion time } \\
\text { - Case conference and journey board process in place }\end{array}$ \\
\hline Workforce & $\begin{array}{l}\text { Team coordination } \\
\text { Different health professional team structures } \\
\text { Perception of respect }\end{array}$ & $\begin{array}{l}\text { - Difficulty in team meeting at an agreed time } \\
\text { - Team structures not uniform } \\
\text { - Some patients and discussion irrelevant to different clinicians } \\
\text { - Power imbalance between disciplines }\end{array}$ \\
\hline Care planning & $\begin{array}{l}\text { Patient factors } \\
\text { Environment } \\
\text { Disrupts routine care }\end{array}$ & $\begin{array}{l}\text { - Uncomfortable for patients } \\
\text { - Too many around bed space } \\
\text { - Competing priorities caring for other patients }\end{array}$ \\
\hline
\end{tabular}

Increased time factor. Patient (+ family) would want more input. [AH2]

Although the importance of interdisciplinary rounds was acknowledged, nurses had concerns about how the rounds would affect other patients, and nurses' ability to provide care for them. Nursing staff considered that the round may limit the time staff had to provide care to their other patients.

Can be time consuming for nurse, depending on time of day and demands of other patients. [N1]

I feel multidisciplinary ward rounds are essential component to patient care, however time often doesn't permit us to be part of the process. [N13]

Allied health clinicians stated interdisciplinary rounds overlap with other processes already in place. These are the journey board and case conference. The former is an electronic whiteboard used for discharge planning and patient flow and located in the ward corridor. ${ }^{16} \mathrm{~A}$ case conference consists of members of the interdisciplinary team meeting in a room to discuss patient goals and treatment plans. A patient can be invited to attend ${ }^{10}$ Allied health professionals questioned if it was necessary to have parallel processes, which they viewed as leading to inefficient use of time.

Is it necessary when we have case conference and journey board already? [AH17]

Overlaps with case conference and journey board meetings. [A18]

\section{Theme Two: Workforce}

Challenges identified with "workforce" were lack of team coordination, structure and respect. Clinicians from all health professions nominated difficulties with team coordination. This was defined as being unable to have attendees present at the same time due to competing priorities, coordinating meal breaks and finding a convenient time for the round. Medical officers and allied health clinicians reported not all patients require interdisciplinary team involvement, and this would affect coordination of the round. One allied health clinician stated that failing to have adequate team coordination could lead to an extended round; attendees arriving late would need to be updated on issues already discussed.

Getting it organized so all can be available at one time. People have to have breaks (tea breaks). Organising could be the biggest problem. [N44]

Can be difficult with getting all staff involved i.e. doctor, nurse, allied health at the same time. [AH1]

Some aspects of care not relevant to all members of multidisciplinary team. [M6]

Team coordination is linked closely with the team structure. Nursing and allied health clinicians raised this as a challenge as each health professional team is structured differently. Teams that are not ward based, but specialty based, resulted in nurses caring for patients from different medical and allied health teams. Nurses were concerned that being involved in the round meant they were not available to other patients and argued increased staffing levels would be required to facilitate their involvement. Allied health clinicians, not ward based but hospital based, reported limited staffing levels that would prevent them from attending all rounds for their patients. They were the only health profession to comment on having to cover multiple wards, therefore specialities. 
All staff can cover half hospital, or all hospital therefore can't do all the rounds. [AH1]

Nurses identified challenges related to a hierarchical workforce. They described this as not being asked their opinion by medical officers which resulted in them not offering their opinion into care planning.

Fear of hierarchy - perhaps my opinion on medical aspect I wouldn't voice to doctors? [N20]

Nursing input not always obtained ... [N25]

\section{Theme Three: Care Planning}

Challenges of "care planning" were more likely to be identified in terms of negative impacts on patients, rather than on clinicians. Challenges resulting from patient factors, environment and routine care provision were identified.

Participants from all health professions were concerned about the effect rounds would have on patients regarding privacy and limited space. Nurses also identified allied health professionals included multiple disciplines, and there may not be the physical space for them at the bedside.

All three professions said the patient would feel overwhelmed and intimidated by having multiple clinicians at the bedside. The physical effect of increasing a patient's confusion could be a side effect. All three professions reported a lack of privacy for patients in a multi-bed room as a concern. This included the inappropriateness of some discussions to have about patients in front of them. An allied heath clinician felt there were already concerns with privacy during other rounding processes such as the journey board due to the communal areas it is undertaken.

Some things not appropriate to discuss in front of patient. [A7]

Confused patients become more confused. [M1]

Overcrowding patient or intimidating patient. [M2]

Disruption to routine care was implied in many responses by all health professionals. Participants were concerned patients would lose therapy and clinical time while staff attended rounds. It was perceived that rounds could disrupt the routine of staff and may impact negatively on patient care provision.

Patients lose their therapy time on ward round days. [M11]
Prevents nurses or other team members from providing other patient care and giving medications. [N35]

Increased time taken to attend which means other patients may miss out on therapy. [A19]

\section{Discussion}

This study investigated the perceptions of medical officers, nurses, and allied health clinicians on the benefits and challenges of IBRs. Our findings add to an emerging knowledge base documenting clinicians' perceptions of rounding processes undertaken on their wards and impacts on patient care, safety and quality. ${ }^{17,18}$

There was a juxtaposition between these benefits and challenges that revealed the complexities of IBRs. This was a similar finding to an earlier study by Merchant and Federman $^{19}$ where reconciling benefits and challenges were identified. The themes of "being on the same page", "focusing on patients", and "holistic care planning" underpinned the ideas of collaboration and improved patient-centred care, that is, benefits to patients. These themes were closely linked through interconnecting key elements centring around improved patient care. The challenges of IBRs were more distinct, logistical barriers of "time", "workforce", and "care planning". Many of the challenging key elements centred around how IBRs affect clinicians, as opposed to patients. For example, the benefit of having all team members present and providing an opinion improved team communication and communication with the patient; yet this was equally a challenge to available time, resulting in a longer rounding process. This idea was summarised by one nurse's comment that IBRs are essential, but time does not allow participation.

Our findings indicate that clinicians want to work in a cohesive interdisciplinary team. IBRs were perceived to facilitate effective interdisciplinary communication, yet some health professionals, for example nurses, feared their opinion would not be valued by the medical hierarchy. This was despite medical officers believing having nurses and allied health clinicians at a round would provide them with additional patient information. These contrasting ideas and perspectives reveal the complex social context and organizational culture than just what is experienced at an IBR. Clinicians are influenced by the hospital context and culture which affect their interdisciplinary attitudes and practice. ${ }^{20}$ Studies have shown challenges around boundaries, such as authority and intradisciplinary standards of conduct, strongly influence health professionals. ${ }^{11,21}$ The responses also show 
the influence medical officers have not only their own identity but that of nurses which contributes to interdisciplinary boundaries. $^{11}$

Despite rounds being considered a cornerstone of patient care planning, ${ }^{2,3}$ nursing and allied health clinicians frequently commented that IBRs prevented them from providing routine care to their patients. A distinction between the planning and provision of care was clearly made. Furthermore, there is a disconnection between how nurses and allied health clinicians see their roles in IBRs. Although rounding processes have evolved, medical officers largely remain the central participants. This may influence the idea that any rounding process involving medical officers is a medical activity. Nursing and allied health professionals commented that they frequently felt unable to contribute, and since their role was that of advocate and not decision-maker, they were not as important as the medical staff. These perceptions are supported by empirical ethnographic research that has shown distinctive participation patterns in care planning and decision-making: doctors talk and other professionals are expected to listen. ${ }^{20}$

For allied health professionals, attending IBRs experienced as doubling up on processes already in place. They likened the journey board and case conferences to IBRs, and therefore IBRs to be an inefficient use of time. As many allied health professionals cover up to half, or even all, the hospital on a given day, attending IBRs is logistically challenging. A negative relationship between efficiency and clinical structures that do not support IBRs can be drawn. Additionally, the physical hospital context is a barrier to efficiency in IBRs for allied health professionals. By way of contrast, the co-location of teams undertaking IBRs is one of the features of higher functioning teams. ${ }^{4,18}$

Our study builds upon that conducted by Gonzalo, Kuperman, Lehman and Haidet ${ }^{3}$ who explored perceptions of IBRs among medical officers and nurses working in internal medicine. The inclusion of allied health clinicians in our study offers a broader clinical perspective on rounding processes and addresses a limitation of the Gonzalo study. Clinicians in our study said interdisciplinary communication and cohesive teamwork were benefits of IBRs. This supports findings from Gonzalo, Kuperman, Lehman and Haidet $^{3}$ when medical officers and nurses ranked interprofessional communication and collaboration as the highest benefits of IBRs. While IBRs have been identified as providing educational opportunities, ${ }^{2}$ no respondents in our study indicated this as either a benefit or a challenge. This was in contrast to the study undertaken by Gonzalo, Kuperman, Lehman and Haidet ${ }^{3}$ who found respondents ranked education during IBRs as a positive function. IBRs are an opportunity to provide patient-centered care, and our findings reflect the desire of health professionals to deliver care within this model. Despite the perceived barriers, the findings suggest clinicians recognise there are greater benefits to IBRs and have a willingness to participate.

This study provided unexpected insights into care coordination. The foundation for successful IBRs requires effective teamwork. Understanding a team's perspectives on barriers enables them to be addressed and facilitate more effective team functioning. Exploring commonalities and differences in perceptions leads to asking if clinicians experience cohesive teamwork, or is this an aspirational goal? All health professionals expressed a desire to work as an interdisciplinary team, yet disparate perceptions of team collaboration challenged this. Medical officers were wanting interdisciplinary input while some nurses and allied health professionals were cautious at how this may be received.

Nursing and allied health clinicians described benefits and challenges from two perspectives. They started by responding from the perspective of working with patients as individual clinicians, to working with patients as a team. Medical officers responded less from an individual perspective and more from being part of an interdisciplinary team.

A high functioning teamwork culture can help interdisciplinary teams deliver safer care. ${ }^{22}$ The differences in the interdisciplinary team's perceptions of IBRs provide a direction to further investigate: what does teamwork mean to different health professionals and disciplines? A literature scan revealed a gap in exploring the processes and experiences of new clinical team members joining IBRs. This leads to asking how these clinicians, and ones in a consultative role, are introduced to IBR processes and expectations. There is an opportunity to further explore how clinicians who are increasingly becoming members of clinical teams, such psychologists and podiatrists, integrate into established rounding processes and practices.

\section{Conclusion}

Collaboration and care coordination will remain a challenge due to the unpredictable nature of the ward environment and processes of the healthcare system. How organizations 
support healthcare teams to manage these challenges will contribute to clinicians planning for these situations. The introduction of IBRs, or any rounding process, needs to be well planned and structured. This will facilitate incorporation of rounds into routine patient care. As patient acuity increases, and lengths of stay decrease, improved effectiveness and efficiency of interdisciplinary collaboration, communication and care planning become bedrock to highquality care.

\section{Ethical Approval}

Northern Sydney Local Health District Human Ethics Committee LNR.13.HAWKE.433.

\section{Disclosure}

The authors report no conflicts of interest in this work.

\section{References}

1. Nedfors K, Borg C, Fagerstro C. Communication with physicians in hospital rounds: an interview with nurses. Nord J Nurs Res. 2015;36 (3):122-127. doi:10.1177/0107408315606633

2. Walton V, Hogden A, Johnson J, Greenfield D. Ward rounds, participants, roles and perceptions: literature review. Int J Health Care Qual Assur. 2016;29(4):364-379. doi:doi:10.1108/JJHCQA-04-2015-0053

3. Gonzalo JD, Kuperman E, Lehman E, Haidet P. Bedside interprofessional rounds: perceptions of benefits and barriers by internal medicine nursing staff, attending physicians, and housestaff physicians. $J$ Hosp Med. 2014;9(10):646-651. doi:10.1002/jhm.2245

4. Bhamidipati VS, Elliott DJ, Justice EM, Belleh E, Sonnad SS, Robinson EJ. Structure and outcomes of interdisciplinary rounds in hospitalized medicine patients: a systematic review and suggested taxonomy. J Hosp Med. 2016;11(7):513-523. doi:10.1002/jhm.2575

5. Körner M. Interprofessional teamwork in medical rehabilitation: a comparison of multidisciplinary and interdisciplinary team approach. Clin Rehabil. 2010;24(8):745-755. doi:10.1177/ 0269215510367538

6. Najafi N, Monash B, Mourad M, et al. Improving attending rounds: qualitative reflections from multidisciplinary providers. Hosp Pract. 2015;43(3):186-190. doi:10.1080/21548331.2015.1043181

7. Walton V, Hogden A, Long JC, Johnson J, Greenfield D. Clinicians' perceptions of rounding processes and effectiveness of clinical communication. J Eval Clin Pract. 2019. doi:10.1111/jep.13248
8. Weller J, Boyd M, Cumin D. Teams, tribes and patient safety: overcoming barriers to effective teamwork in healthcare. Postgrad Med J. 2014;90(1061):149-154. doi:10.1136/postgradmedj-2012-131168

9. O'Leary KJ, Killarney A, Hansen LO, et al. Effect of patient-centred bedside rounds on hospitalised patients' decision control, activation and satisfaction with care. BMJ Qual Saf. 2016;25(12):921-928. doi:10.1136/bmjqs-2015-004561

10. Ward SA, Workman B. Multidisciplinary teamwork. In: Caplan G, editor. Geriatric Medicine an Introduction. Melbourne: IP Communications, Pty. Ltd.; 2014:30-46.

11. Liberati EG, Gorli M, Scaratti G. Invisible walls within multidisciplinary teams: disciplinary boundaries and their effects on integrated care. Soc Sci Med. 2016;150:31-39. doi:10.1016/j.socscimed. 2015.12.002

12. Enslev Jensen B, Found PA, Williams SJ, Walley P. Improving the efficiency and effectiveness of ward rounds. IJQSS. 2016;8 (3):279-297. doi:doi:10.1108/IJQSS-05-2016-0039

13. Stein J, Payne C, Methvin A, et al. Reorganizing a hospital ward as an accountable care unit. $J$ Hosp Med. 2015;10(1):36-40. doi:10.1002/jhm.2284

14. Bazeley P. Qualitative Data Analaysis Practical Strategies. London: Sage; 2013.

15. Schwandt TA, Lincoln YS, Guba EG. Judging interpretations: but is it rigorous? Trustworthiness and authenticity in naturalistic evaluation. New Dir Eval. 2007;2007(114):11-25. doi:10.1002/ ev. 223

16. Clark KW, Moller S, O’Brien L. Electronic patient journey boards a vital piece of the puzzle in patient flow. Aust Health Rev. 2014;38 (3):259-264. doi:10.1071/AH13192

17. Clay-Williams R, Plumb J, Luscombe GM, et al. Improving teamwork and patient outcomes with daily structured interdisciplinary bedside rounds: a multimethod evaluation. J Hosp Med. 2018;13 (5):311-317. doi:10.12788/jhm.2850

18. O'Leary KJ, Johnson JK, Auerbach AD. Do interdisciplinary rounds improve patient outcomes? Only if they improve teamwork. J Hosp Med. 2016;11(7):524-525. doi:10.1002/jhm.2587

19. Merchant NB, Federman DG. Bedside rounds valued but not preferred: perceptions of internal medicine residents and attending physicians in a diverse academic training program. South Med J. 2017;110(8):531-537. doi:10.14423/SMJ.0000000000000689

20. Nugus P, Greenfield D, Travaglia J, Westbrook J, Braithwaite J. How and where clinicians exercise power: interprofessional relations in health care. Soc Sci Med. 2010;71(5):898-909. doi:10.1016/j. socscimed.2010.05.029

21. Bate P. Changing the culture of a hospital: from hierarchy to networked community. Public Adm. 2000;78(3):485. doi:10.1111/14679299.00215

22. Körner M, Bütof S, Müller C, Zimmermann L, Becker S, Bengel J. Interprofessional teamwork and team interventions in chronic care: a systematic review. J Interprof Care. 2016;30(1):15-28. doi:10. $3109 / 13561820.2015 .1051616$

\section{Publish your work in this journal}

The Journal of Multidisciplinary Healthcare is an international, peerreviewed open-access journal that aims to represent and publish research in healthcare areas delivered by practitioners of different disciplines. This includes studies and reviews conducted by multidisciplinary teams as well as research which evaluates the results or conduct of such teams or healthcare processes in general. The journal covers a very wide range of areas and welcomes submissions from practitioners at all levels, from all over the world. The manuscript management system is completely online and includes a very quick and fair peer-review system. Visit http://www.dovepress.com/testimonials. php to read real quotes from published authors. 